\title{
Robust Active Disturbance Rejection Control Approach to Maximize Energy Capture in Variable-Speed Wind Turbines
}

\author{
Horacio Coral-Enriquez, John Cortés-Romero, and Germán A. Ramos \\ Universidad Nacional de Colombia, Departamento de Ingeniería Eléctrica y Electrónica, Carrera 45 No. 26-85, Bogotá, Colombia \\ Correspondence should be addressed to Horacio Coral-Enriquez; hacorale@unal.edu.co
}

Received 19 April 2013; Accepted 30 May 2013

Academic Editor: Ming-Hung Hsu

Copyright ( 2013 Horacio Coral-Enriquez et al. This is an open access article distributed under the Creative Commons Attribution License, which permits unrestricted use, distribution, and reproduction in any medium, provided the original work is properly cited.

\begin{abstract}
This paper proposes an alternative robust observer-based linear control technique to maximize energy capture in a $4.8 \mathrm{MW}$ horizontal-axis variable-speed wind turbine. The proposed strategy uses a generalized proportional integral (GPI) observer to reconstruct the aerodynamic torque in order to obtain a generator speed optimal trajectory. Then, a robust GPI observer-based controller supported by an active disturbance rejection (ADR) approach allows asymptotic tracking of the generator speed optimal trajectory. The proposed methodology controls the power coefficient, via the generator angular speed, towards an optimum point at which power coefficient is maximum. Several simulations (including an actuator fault) are performed on a $4.8 \mathrm{MW}$ wind turbine benchmark model in order to validate the proposed control strategy and to compare it to a classical controller. Simulation and validation results show that the proposed control strategy is effective in terms of power capture and robustness.
\end{abstract}

\section{Introduction}

The use of wind energy has a history of over a hundred years. Its applications include agriculture, milling, water pumping, and power production. In the 1970s, this technology started developing as an experimental technology. Nowadays, the conversion of wind energy into electrical energy by wind turbines is a mature technology that exhibits the highest growth rates among the renewable energy sources [1] and can be considered as the most promising option for replacing a significant part of the electricity produced by conventional sources [2].

The main objective of wind turbines is to convert efficiently wind energy into electrical power. There are wind turbines available in a different number of configurations (vertical axis, horizontal axis, fixed speed, variable speed, etc.). The most used type for large-scale power production is the variable-speed horizontal-axis wind turbine (HAWT) with a two- or three-blade rotor [3]. HAWTs are commonly equipped with blade-pitch actuators, generator torque control and many sensors for use in real time control [4]. HAWTs operate in different control regions, and the region considered in the present work is a low-to-medium wind speeds operation (also called partial load operation or operation in region 2) where the main objective is to extract the maximum power from the wind.

Modern wind turbines are machines that require big efforts when maximizing wind energy capture, not only because of their highly nonlinear aerodynamics, but also because of the high efficiency required even when model uncertainties, external perturbations, or system faults are present. As a consequence, the efficiency of both power capture and power generation is strongly dependent on the selected control method [5]. This situation provides a motivation to consider new alternative control techniques that improve the performance of HAWT without any structural change.

A large number of control schemes to find the best way of solving the energy capture maximization problem for wind turbines at low-to-medium wind speeds have been proposed (see, e.g., [6-12]). The control techniques range from standard torque control [6], disturbance tracking control [7], maximum power point tracking [8], and aerodynamic torque feedforward [9] to complex nonlinear strategies [5, 10-12]. Most of these techniques deal with the wind turbine complexity using linearization techniques or nonlinear control. Following a different approach, some of the active-disturbancerejection- (ADR-) based techniques allow linear control 


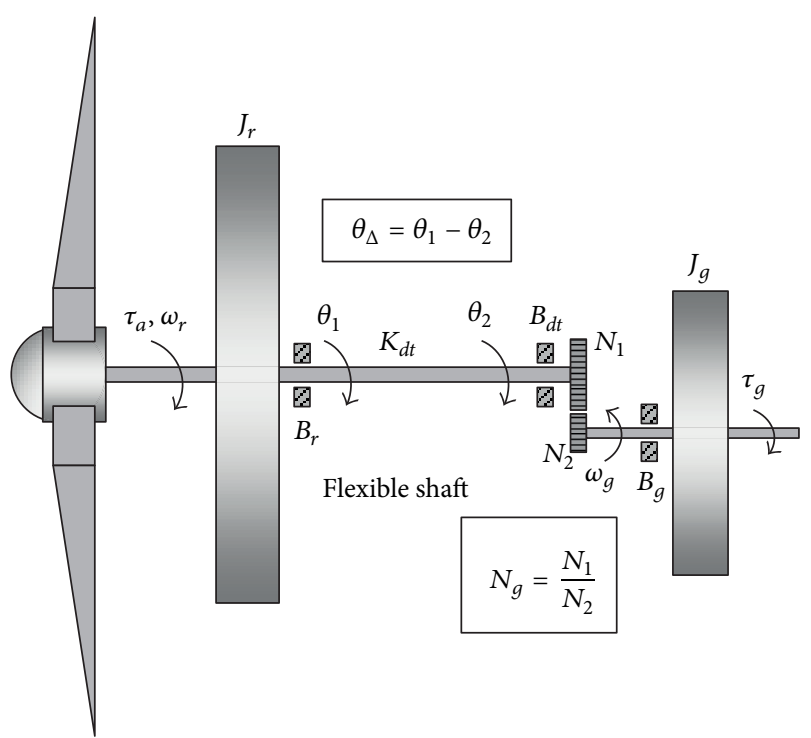

(a)

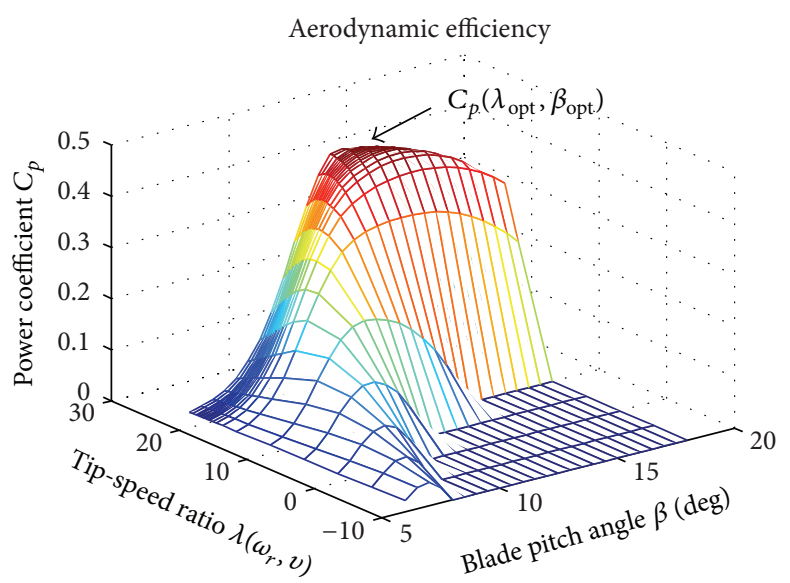

(b)

FIGURE 1: Mechanical and aerodynamical characteristics of the wind turbine.

solutions for some class of uncertain complex nonlinear systems and could offer a linear, simpler, and robust solution to the wind energy capture maximization problem. This is the case of the ADR philosophy-based technique called generalized proportional integral (GPI) Control [13] and its GPI observer-based control extensions $[14,15]$.

Generalized proportional integral (GPI) control technique was started in 2000 by Fliess et al. $[13,16]$ and involves in its design the active rejection of disturbances. The dual counterpart of the generalized proportional integral controller, called GPI observer, was introduced in [15], in the context of sliding mode observers for flexible robotics systems. The nonsliding version appears in [14] applied to chaotic systems synchronization. The GPI control strategies have been adapted, extended, and applied successfully in areas other than wind energy, such as induction motor control [17], chaotic systems control [18], and power converters control [19]. Therefore, it is interesting to adapt, evaluate, and determine the scope of this control method in partial load wind turbine operation.

GPI observer-based control of nonlinear uncertain systems is very much related to methodologies known as disturbance accommodation control (DAC) and active disturbance rejection control (ADRC). These approaches deal with the problem of cancelling, from the controller's actions, endogenous and exogenous unknown additive disturbance inputs affecting the system. Perturbation effects are made available via a suitable linear or nonlinear estimation. The reader is invited to read the works by Johnson [20], Han [21], and Gao et al. [22, 23].

This work presents an alternative linear control technique based on GPI observers to maximize wind energy capture in variable-speed wind turbines operating at partial load. The proposed strategy uses a GPI observer to reconstruct the aerodynamic torque in order to provide a generator speed optimal trajectory to a robust GPI observer-based controller that regulates the power coefficient, via the generator torque, towards an optimum point at which the power coefficient is maximum. It is expected that the proposed GPI observerbased control technique adds robustness to the system and solves the control problem through linear active estimation and rejection of nonlinearities and perturbations of the wind energy conversion system (WECS).

This paper is organized as follows. In Section 2, a wind turbine dynamic model for control purposes is presented. Section 3 formulates the control problem and presents the proposed methodology to solve it. Section 4 describes the benchmark and the tests model used to validate the proposed methodology. Section 5 presents the simulations and validations results of the proposed control strategy. Finally, Section 6 contains the conclusions and suggestions for further work.

\section{Wind Turbine Model}

Consider a wind turbine represented by a two-mass mechanical system as shown in Figure 1(a), where the aerodynamic power captured by the rotor is given by [24]

$$
P_{a}(t)=\frac{1}{2} \rho \pi R^{2} C_{p}(\lambda, \beta) v(t)^{3}
$$

where $R$ is the rotor radius, $\rho$ is the air density, $v(t)$ is the wind velocity, and $C_{p}(\lambda, \beta)$ is the power coefficient which denotes the aerodynamic efficiency of the wind energy conversion system. The power coefficient curve $C_{p}(\lambda, \beta)$ of the wind turbine considered in this work is shown in Figure 1(b). This curve was taken from the benchmark model published in [25]. The power coefficient $C_{p}(\lambda, \beta)$ depends on both 
the blade pitch angle $\beta$ and the tip-speed ratio $\lambda$. The latter is defined as

$$
\lambda=\frac{\omega_{r}(t) R}{v(t)},
$$

where $\omega_{r}(t)$ is the rotor angular speed. The aerodynamic power $P_{a}(t)$ can also be expressed in function of both the aerodynamic torque $\tau_{a}(t)$ and the rotor speed $\omega_{r}(t)$ as follows [24]:

$$
P_{a}(t)=\omega_{r}(t) \tau_{a}(t) .
$$

The aerodynamic torque $\tau_{a}(t)$ is given by

$$
\tau_{a}(t)=\frac{1}{2} \rho \pi R^{3} C_{q}(\lambda, \beta) v(t)^{2},
$$

where $C_{q}$ is the torque coefficient and is defined as follows:

$$
C_{q}(\lambda, \beta)=\frac{C_{p}(\lambda, \beta)}{\lambda} .
$$

The mechanical system of the wind turbine is modeled using Newton's laws, and the following system is derived [25]:

$$
\begin{gathered}
\frac{d}{d t} \mathbf{x}_{\mathbf{w t}}(t)=\mathbf{A}_{\mathbf{w t}} \mathbf{x}_{\mathbf{w t}}(t)+\mathbf{B}_{\mathbf{w t}} \tau_{g}(t)+\mathbf{F}_{\mathbf{w t}} \tau_{a}(t), \\
y(t)=\mathbf{C}_{\mathbf{w t}} \mathbf{x}_{\mathbf{w t}}(t)
\end{gathered}
$$

with

$$
\begin{aligned}
\mathbf{x}_{\mathbf{w t}}(t)=\left[\begin{array}{c}
\omega_{r}(t) \\
\omega_{g}(t) \\
\theta_{\Delta}(t)
\end{array}\right], \\
\mathbf{A}_{\mathbf{w t}}=\left[\begin{array}{ccc}
\frac{-B_{d t}-B_{r}}{J_{r}} & \frac{B_{d t}}{J_{r} N_{g}} & \frac{-K_{d t}}{J_{r}} \\
\frac{B_{d t} \eta_{d t}}{J_{g} N_{g}} & \frac{-\left(B_{g}+\left(B_{d t} \eta_{d t} / N_{g}^{2}\right)\right)}{J_{g}} & \frac{K_{d t} \eta_{d t}}{J_{g} N_{g}} \\
1 & \frac{-1}{N_{g}} & 0 \\
\mathbf{B}_{\mathbf{w t}} & {\left[\begin{array}{c}
0 \\
\frac{-1}{J_{g}} \\
0
\end{array}\right],} \\
\frac{1}{J_{r}} \\
0 \\
0
\end{array}\right], \quad \mathbf{F}_{\mathbf{w t}}=\left[\begin{array}{lll}
0 & 1
\end{array}\right],
\end{aligned}
$$

where $\omega_{g}(t)$ is the generator side angular speed, $\theta_{\Delta}(t)$ is the torsion angle of the drive train, $J_{r}$ is the moment of inertia of the low speed shaft, $J_{g}$ is the moment of inertia of the high speed shaft, $K_{d t}$ is the torsion stiffness of the drive train, $B_{d t}$ is the torsion damping coefficient of the drive train, $B_{r}$ is the viscous friction of the low speed shaft, $B_{g}$ is the viscous friction of the high speed shaft, $N_{g}$ is the gear ratio, $\eta_{d t}$ is the efficiency of the drive train, and $\tau_{g}(t)$ is the generator torque. [25]

The power converter and generator dynamics are given by

$$
\begin{gathered}
\frac{d}{d t} \tau_{g}(t)=-\alpha_{\mathrm{gc}} \tau_{g}(t)+\alpha_{\mathrm{gc}} \tau_{g, \mathrm{ref}}(t) \\
P_{g}(t)=\eta_{g} \omega_{g}(t) \tau_{g}(t)
\end{gathered}
$$

respectively, where $\tau_{g \text {,ref }}(t)$ is the desired generator torque (control input), $P_{g}(t)$ is the produced power by the generator, $\alpha_{\mathrm{gc}}$ denotes the dynamic coefficient of the generator/converter, and $\eta_{g}$ is the generator efficiency.

\section{Active Disturbance Rejection Design for Wind Turbine Control}

The following assumptions in relation to the system (1)-(9) are stated.

Assumption 1. All the parameters of the WECS are known.

Assumption 2. The pair $\left(\mathbf{A}_{\mathrm{wt}}, \mathbf{C}_{\mathbf{w t}}\right)$ is completely observable.

Assumption 3. The generator angular speed $\omega_{g}(t)$ as well as the generated generator torque $\tau_{g}(t)$ is available to be used in the control system.

Assumption 4. For sufficiently large positive integer $p$, the disturbance input $\tau_{a}(t)$ exhibits uniformly absolute bounded time derivative of order $p$. This condition assures the existence of an unknown but finite constant, $K_{\tau_{a}}$, such that $\sup _{t \geq 0}\left|\tau_{a}^{(p)}(t)\right| \leq K_{\tau_{a}}$.

3.1. Problem Formulation. For a partial load-operating regime (operation in region 2), the main control objective is the maximization of wind power capture. This objective has a strong relation with the wind turbine power coefficient curve $C_{p}(\lambda, \beta)$, which has a unique maximum point that corresponds to the optimal capture of the wind power:

$$
C_{p}\left(\lambda_{\mathrm{opt}}, \beta_{\mathrm{opt}}\right)=C_{p_{\mathrm{opt}}},
$$

where

$$
\lambda_{\mathrm{opt}}=\frac{\omega_{g_{\mathrm{opt}}}(t) R}{N_{g} v(t)} .
$$

Accordingly, in order to maximize wind power capture, the blade pitch angle $\beta$ is fixed to its optimal value $\beta_{\text {opt }}$, and in order to maintain $\lambda$ at its optimal value $\lambda_{\text {opt }}$, the generator speed must be adjusted to track the optimal reference $\omega_{g_{\text {opt }}}(t)$, given by

$$
\omega_{g_{\mathrm{opt}}}(t)=\frac{N_{g} \lambda_{\mathrm{opt}}}{R} v(t)
$$

Then, it is desired to force the output $\omega_{g}(t)$ to accurately track the given trajectory $\omega_{g_{\text {opt }}}(t)$, independently of the aerodynamic torque input and possible unmodeled perturbation inputs in the WECS, using the desired generator torque $\tau_{g, \text { ref }}(t)$ as the control input and the generator angular speed $\omega_{g}(t)$ as the feedback signal.

3.2. GPI Observer Design for Aerodynamic Torque Estimation. In order to obtain the optimal reference $\omega_{g_{\text {opt }}}(t),(4),(5)$, and 
(12) are combined. The wind velocity $v(t)$ is easily represented as a function of $\tau_{a}(t)$ and $C_{p}$ using (4) and (5):

$$
v(t)=\sqrt{\frac{2 \lambda \tau_{a}(t)}{\rho \pi R^{3} C_{p}(\lambda, \beta)}} .
$$

Then, by replacing (13) in (12), setting $\lambda$ and $C_{p}$ to its optimal values, and changing $\tau_{a}(t)$ to its estimated version $\widehat{\tau}_{a}(t)$, the following expression is obtained for the optimal reference trajectory:

$$
\widehat{\omega}_{g_{\mathrm{opt}}}(t)=\frac{N_{g} \lambda_{\mathrm{opt}}}{R} \sqrt{\frac{2 \lambda_{\mathrm{opt}} \widehat{\tau}_{a}(t)}{\rho \pi R^{3} C_{p_{\mathrm{opt}}}}} .
$$

According to (14), it is necessary to estimate the aerodynamic torque $\tau_{a}(t)$. For that purpose, an extended Luenberger-like linear observer is developed, here referred as GPI observer. The proposed observer uses an approximated internal model of the unknown input disturbance to compose an augmented model for the plant and the disturbance input. Inherent to this kind of observer, a state estimation is also provided. This estimation will be used in the establishment of the GPI observerbased control for $\omega_{g_{\text {opt }}}(t)$ tracking.

Given a positive integer $p$, the unknown input perturbation $\tau_{a}(t)$ can be modeled by the approximation of its internal model given by

$$
\frac{d^{p} \tau_{a}(t)}{d t^{p}} \approx 0
$$

Consider the following disturbance states, related to (15):

$$
\mathbf{x}_{\mathbf{d}}(t)=\left[\begin{array}{lllll}
\tau_{a}(t) & \dot{\tau}_{a}(t) & \cdots & \tau_{a}^{(p-2)}(t) & \tau_{a}^{(p-1)}(t)
\end{array}\right]^{T}
$$

where its corresponding dynamics is given by

$$
\begin{gathered}
\frac{d}{d t} \mathbf{x}_{\mathbf{d}}(t)=\mathbf{A}_{\mathbf{d}} \mathbf{x}_{\mathbf{d}}(t)+\mathbf{B}_{\mathbf{d}} \tau_{a}^{(p)}(t), \\
\tau_{a}(t)=\mathbf{C}_{\mathbf{d}} \mathbf{x}_{\mathbf{d}}(t)
\end{gathered}
$$

with

$$
\begin{gathered}
\mathbf{A}_{\mathbf{d}}=\left[\begin{array}{ccccc}
0 & 1 & 0 & \cdots & 0 \\
0 & 0 & 1 & \cdots & 0 \\
\vdots & \vdots & \vdots & \ddots & \vdots \\
0 & 0 & 0 & \cdots & 1 \\
0 & 0 & 0 & \cdots & 0
\end{array}\right], \\
\mathbf{B}_{\mathbf{d}}=\left[\begin{array}{c}
0 \\
0 \\
\vdots \\
0 \\
1
\end{array}\right], \quad \mathbf{C}_{\mathbf{d}}=\left[\begin{array}{lllll}
1 & 0 & 0 & \cdots & 0
\end{array}\right],
\end{gathered}
$$

where $\mathbf{x}_{\mathbf{d}}(t) \in \mathbb{R}^{p \times 1}, \mathbf{A}_{\mathbf{d}} \in \mathbb{R}^{p \times p}, \mathbf{B}_{\mathbf{d}} \in \mathbb{R}^{p \times 1}$, and $\mathbf{C}_{\mathbf{d}} \in \mathbb{R}^{1 \times p}$.
Now, the disturbance states $\mathbf{x}_{\mathbf{d}}(t)$ can be added to the system state vector $\mathbf{x}_{\mathrm{wt}}(t)$ to form the following augmented system:

$$
\begin{gathered}
\frac{d}{d t} \mathbf{x}(t)=\mathbf{A} \mathbf{x}(t)+\mathbf{B} \tau_{g}(t)+\mathbf{B}_{\mathbf{a}} \tau_{a}^{(p)}(t), \\
y(t)=\mathbf{C} \mathbf{x}(t)
\end{gathered}
$$

with

$$
\begin{gathered}
\mathbf{x}(t)=\left[\begin{array}{c}
\mathbf{x}_{\mathbf{w t}}(t) \\
\mathbf{x}_{\mathbf{d}}(t)
\end{array}\right], \quad \mathbf{A}=\left[\begin{array}{cc}
\mathbf{A}_{\mathbf{w t}} & \mathbf{F}_{\mathbf{w t}} \mathbf{C}_{\mathbf{d}} \\
\mathbf{0} & \mathbf{A}_{\mathbf{d}}
\end{array}\right], \\
\mathbf{B}=\left[\begin{array}{c}
\mathbf{B}_{\mathbf{w t}} \\
\mathbf{0}
\end{array}\right], \quad \mathbf{B}_{\mathbf{a}}=\left[\begin{array}{c}
\mathbf{0} \\
\mathbf{B}_{\mathbf{d}}
\end{array}\right], \quad \mathbf{C}=\left[\begin{array}{ll}
\mathbf{C}_{\mathbf{w t}} & \mathbf{0}
\end{array}\right],
\end{gathered}
$$

where $\mathbf{x}(t) \in \mathbb{R}^{(p+3) \times 1}, \mathbf{A} \in \mathbb{R}^{(p+3) \times(p+3)}, \mathbf{B}, \mathbf{B}_{\mathbf{a}} \in \mathbb{R}^{(p+3) \times 1}$, and $\mathbf{C} \in \mathbb{R}^{1 \times(p+3)}$.

The next step is to design a GPI observer for the composite system in (19) regarding the approximated internal model given in (15). The estimated augmented state vector $\mathbf{x}(t)$ contains a real-time estimate of $\mathbf{x}_{\mathbf{d}}(t)$, which is used along with $\mathbf{C}_{\mathbf{d}}$ to recover $\tau_{a}(t)$.

Remark 5. ADR-GPI observer-based controllers use an internal model approximation of the perturbation functions to reconstruct and reject the perturbations. Under this disturbance model approximation setting, several authors have applied it to different areas. Parker and Johnson used a firstorder perturbation approximation to model wind speed perturbations in a wind turbine operating in region 3 [26]. Freidovich and Khalil [27] used a first-order perturbation model approximation to estimate the model uncertainty and disturbance on a nonlinear system. Zhao and Gao also used a firstorder internal model disturbance approximation to estimate the resonance in two-inertia systems [28] and a first- and second-order approximation to estimate the nonlinearities of an actuator [29]. Zheng et al. also used disturbance model approximation applied to disturbance decoupling control [30].

Remark 6. The parameter $p$ is related to the complexity of the signal to estimate, as in the case of Taylor polynomial approximation. A first-order perturbation model approximation means that the internal model approximation is capable to converge towards a constant disturbance. Equation (15) is a more generalized extension of the internal model perturbation function which provides extra information and increases the ability to track different types of disturbances. For example, $p=2$ allows convergence to a disturbance with a constant derivative, $p=3$ allows convergence to a disturbance with a constant acceleration, and so forth.

Theorem 7. Supposing that Assumptions 1-4 are valid, for the augmented WECS represented by (19) and (20), the following GPI observer is proposed:

$$
\frac{d}{d t} \widehat{\mathbf{x}}(t)=\mathbf{A} \widehat{\mathbf{x}}(t)+\mathbf{B} \tau_{g}(t)+\boldsymbol{\alpha}(y-\mathbf{C} \widehat{\mathbf{x}}(t)),
$$


where $\widehat{\mathbf{x}}(t) \quad=\quad\left[\begin{array}{lll}\widehat{\mathbf{x}}_{\mathbf{w t}}(t)^{\mathrm{T}} & \widehat{\mathbf{x}}_{\mathbf{d}}(t)^{\mathrm{T}}\end{array}\right]^{T}=$

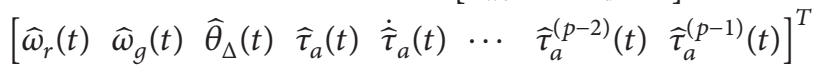
is the augmented system state estimation and $\boldsymbol{\alpha}=$ $\left[\begin{array}{lllll}\alpha_{p+3} & \alpha_{p+2} & \cdots & \alpha_{2} & \alpha_{1}\end{array}\right]^{T}$ are the observer gains. The GPI observer (21) asymptotically and exponentially reconstructs the system states $\omega_{r}(t), \omega_{g}(t), \theta_{\Delta}(t)$, and the perturbation inputs $\tau_{a}(t), \dot{\tau}_{a}(t), \ldots, \tau_{a}^{(p-1)}$ forcing the state estimation error $\widetilde{\mathbf{e}}_{\mathbf{x}}(t)=$ $\mathbf{x}(t)-\widehat{\mathbf{x}}(t)$ to converge towards the interior of a disk centered in the origin of the corresponding estimation error phase space, as long as the set of coefficients $\left\{\alpha_{p+3}, \ldots, \alpha_{2}, \alpha_{1}\right\}$ is chosen in such $a$ way that the characteristic polynomial defined by

$$
\operatorname{det}(s \mathbf{I}-\mathbf{A}+\boldsymbol{\alpha} \mathbf{C})=0
$$

is a Hurwitz polynomial, with roots located to the left of the imaginary axis of the complex plane.

Proof. By subtracting the proposed observer (21) from the augmented system state equation (19), the following estimation error dynamics is obtained:

$$
\dot{\tilde{\mathbf{e}}}_{\mathbf{x}}(t)=(\mathbf{A}-\boldsymbol{\alpha} \mathbf{C}) \widetilde{\mathbf{e}}_{\mathbf{x}}(t)+\mathbf{B}_{\mathbf{a}} \tau_{a}^{(p)}(t)=\mathbf{A}_{\mathbf{a}} \widetilde{\mathbf{e}}_{\mathbf{x}}(t)+\mathbf{B}_{\mathbf{a}} \tau_{a}^{(p)}(t),
$$

where the eigenvalues of $\mathbf{A}_{\mathbf{a}}=(\mathbf{A}-\boldsymbol{\alpha} \mathbf{C})$ can be placed as desired by selecting the gain vector $\boldsymbol{\alpha}$.

In order to obtain an ultimate bound for $\widetilde{\mathbf{e}}_{\mathbf{x}}(t)$, let $\mathbf{Q} \epsilon$ $\mathbb{R}^{(p+3) \times(p+3)}$ be a constant, positive definite symmetric matrix. The proper stable character of the matrix $\mathbf{A}_{\mathbf{a}}$ implies the existence of a positive definite matrix $\mathbf{P} \in$ $\mathbb{R}^{(p+3) \times(p+3)}$ such that $\mathbf{A}_{\mathbf{a}}^{\mathbf{T}} \mathbf{P}+\mathbf{P A}_{\mathbf{a}}=-\mathbf{Q}$. Consider the Lyapunov function candidate $V\left(\widetilde{\mathbf{e}}_{\mathbf{x}}\right)=(1 / 2) \widetilde{\mathbf{e}}_{\mathbf{x}}^{\mathbf{T}}(t) \mathbf{P} \widetilde{\mathbf{e}}_{\mathbf{x}}(t)$. The time derivative of $V$ satisfies

$$
\dot{V}\left(\widetilde{\mathbf{e}}_{\mathbf{x}}, t\right)=\frac{1}{2}\left[\widetilde{\mathbf{e}}_{\mathbf{x}}^{\mathrm{T}}(-\mathbf{Q}) \widetilde{\mathbf{e}}_{\mathbf{x}}\right]+\mathbf{B}_{\mathbf{a}}^{\mathrm{T}} \mathbf{P} \widetilde{\mathbf{e}}_{\mathbf{x}} \tau_{a}^{(p)}(t) .
$$

For $Q=I$, that is, an identity matrix, $\dot{V}\left(\widetilde{\mathbf{e}}_{\mathbf{x}}, t\right)$ satisfies

$$
\begin{aligned}
\dot{V}\left(\widetilde{\mathbf{e}}_{\mathbf{x}}, t\right) & =\frac{1}{2}\left[\widetilde{\mathbf{e}}_{\mathbf{x}}^{\mathrm{T}}(-\mathbf{Q}) \widetilde{\mathbf{e}}_{\mathbf{x}}\right]+\mathbf{B}_{\mathbf{a}}^{\mathrm{T}} \mathbf{P} \widetilde{\mathbf{e}}_{\mathbf{x}} \tau_{a}^{(p)}(t) \\
& \leq-\frac{1}{2}\left\|\widetilde{\mathbf{e}}_{\mathbf{x}}\right\|_{2}^{2}+\left\|\mathbf{B}_{\mathbf{a}}^{\mathrm{T}}\right\|_{2}\|\mathbf{P}\|_{2}\left\|\widetilde{\mathbf{e}}_{\mathbf{x}}\right\|_{2} K_{\tau_{a}}<0 .
\end{aligned}
$$

Given that $\left\|\mathbf{B}_{\mathbf{a}}^{\mathrm{T}}\right\|_{2}=1$ and according to $(25), \dot{V}\left(\widetilde{\mathbf{e}}_{\mathbf{x}}, t\right)$ is strictly negative if

$$
\left\|\widetilde{\mathbf{e}}_{\mathbf{x}}\right\|_{2}>2 K_{\tau_{a}}\|\mathbf{P}\|_{2}
$$

Therefore, $\dot{V}\left(\widetilde{\mathbf{e}}_{\mathbf{x}}, t\right)$ is strictly negative outside the following disc:

$$
D_{x}=\left\{\widetilde{\mathbf{e}}_{\mathbf{x}}(t) \in \mathbb{R}^{p+3},\left\|\widetilde{\mathbf{e}}_{\mathbf{x}}\right\|_{2} \leq 2 K_{\tau_{a}}\|\mathbf{P}\|_{2}\right\}
$$

Consequently, a uniform ultimate bounded (UUB) result was obtained regarding the estimation error phase variables $\widetilde{\mathbf{e}}_{\mathbf{x}}(t)$.
Remark 8. GPI observers are bandwidth limited by the roots location of the estimation error characteristic polynomial. Generally, the larger the observer bandwidth is, the more accurate the estimation will be. However, a large observer bandwidth will increase noise sensitivity. Then, the selection of the roots of the estimation error characteristic polynomial affects the bandwidth of the GPI observer and also the influence of measurement noises on the estimations. Therefore, GPI observers are usually tuned in a compromise between disturbance estimation performance (set by the internal model approximation degree) and noise sensitivity.

3.3. Robust GPI Observer-Based Control Design for Energy Capture Maximization. Based on (6), the generator angular speed $\omega_{g}(t)$ satisfies the following dynamics:

$$
\begin{aligned}
\frac{d \omega_{g}(t)}{d t}= & \frac{B_{d t} \eta_{d t}}{J_{g} N_{g}} \omega_{r}(t)-\frac{B_{g}+\left(B_{d t} \eta_{d t} / N_{g}^{2}\right)}{J_{g}} \omega_{g}(t) \\
& +\frac{K_{d t} \eta_{d t}}{J_{g} N_{g}} \theta_{\Delta}(t)-\frac{1}{J_{g}} \tau_{g}(t) .
\end{aligned}
$$

Then, by reorganizing and lumping together some terms of (28), the following simplified system (typical of the ADR philosophy) can be obtained:

$$
\dot{\omega}_{g}(t)=\kappa \tau_{g}(t)+\Delta(t)+\varphi(t)
$$

with

$$
\begin{gathered}
\kappa=-\frac{1}{J_{g}}, \\
\varphi(t)=\frac{B_{d t} \eta_{d t}}{J_{g} N_{g}} \omega_{r}(t)-\frac{B_{g}+\left(B_{d t} \eta_{d t} / N_{g}^{2}\right)}{J_{g}} \omega_{g}(t) \\
+\frac{K_{d t} \eta_{d t}}{J_{g} N_{g}} \theta_{\Delta}(t),
\end{gathered}
$$

where $\kappa$ is a known constant, $\varphi(t)$ is a state dependent input perturbation, and $\Delta(t)$ is an input perturbation function that lumps together all the uncertainty associated to the system. The perturbation $\Delta(t)$ contains the rest of the system dynamics (actuator), including some unmodeled dynamics, disturbances of additive nature, actuator faults, parameter variations, and nonlinear effects of the WECS.

In relation to the simplified system (29), the following assumption is stated.

Assumption 9. For a sufficiently large positive integer $m$, the disturbance input $\Delta(t)$ exhibits a uniformly absolute bounded time derivative of order $m$. This condition assures the existence of an unknown but finite constant, $K_{\Delta}$, such that $\sup _{t \geq 0}\left|\Delta^{(m)}(t)\right| \leq K_{\Delta}$.

Assumption 10. The unknown input perturbation $\Delta(t)$ can be modeled by the approximation of its internal model given by

$$
\frac{d^{m} \Delta(t)}{d t^{m}} \approx 0
$$


Consider the following disturbance states, related to (31),

$$
\mathbf{x}_{\Delta}(t)=\left[\begin{array}{lllll}
\Delta(t) & \dot{\Delta}(t) & \cdots & \Delta^{(m-2)}(t) & \Delta^{(m-1)}(t)
\end{array}\right]^{T},
$$

where their corresponding dynamics is given by:

$$
\begin{gathered}
\frac{d}{d t} \mathbf{x}_{\Delta}(t)=\mathbf{A}_{\Delta} \mathbf{x}_{\Delta}(t)+\mathbf{B}_{\Delta} \Delta^{(m)}(t), \\
\Delta(t)=\mathbf{C}_{\Delta} \mathbf{x}_{\Delta}(t)
\end{gathered}
$$

with

$$
\begin{gathered}
\mathbf{A}_{\Delta}=\left[\begin{array}{ccccc}
0 & 1 & 0 & \cdots & 0 \\
0 & 0 & 1 & \cdots & 0 \\
\vdots & \vdots & \vdots & \ddots & \vdots \\
0 & 0 & 0 & \cdots & 1 \\
0 & 0 & 0 & \cdots & 0
\end{array}\right], \quad \mathbf{B}_{\Delta}=\left[\begin{array}{c}
0 \\
0 \\
\vdots \\
0 \\
1
\end{array}\right] \\
\mathbf{C}_{\Delta}=\left[\begin{array}{lllll}
1 & 0 & 0 & \cdots & 0
\end{array}\right],
\end{gathered}
$$

where $\mathbf{x}_{\Delta}(t) \in \mathbb{R}^{m \times 1}, \mathbf{A}_{\Delta} \in \mathbb{R}^{m \times m}, \mathbf{B}_{\Delta} \in \mathbb{R}^{m \times 1}$, and $\mathbf{C}_{\Delta} \in$ $\mathbb{R}^{1 \times m}$.

Then, it is possible to augment the simplified system in (29) with the unknown input perturbation state vector $\mathbf{x}_{\Delta}(t)$; thus,

$$
\begin{gathered}
\frac{d}{d t} \mathbf{x}_{c}(t)=\mathbf{A}_{c} \mathbf{x}_{c}(t)+\mathbf{B}_{c 1} \tau_{g}(t)+\mathbf{B}_{c 2} \varphi(t)+\mathbf{B}_{c 3} \Delta^{(m)}(t), \\
y(t)=\mathbf{C}_{c} \mathbf{x}_{c}(t)
\end{gathered}
$$

with

$$
\begin{aligned}
\mathbf{x}_{c}(t)=\left[\begin{array}{c}
\omega_{g}(t) \\
\mathbf{x}_{\Delta}(t)
\end{array}\right], & \mathbf{A}_{c}=\left[\begin{array}{ll}
0 & \mathbf{C}_{\Delta} \\
0 & \mathbf{A}_{\Delta}
\end{array}\right], \quad \mathbf{B}_{c 1}=\left[\begin{array}{c}
\kappa \\
\mathbf{0}
\end{array}\right], \\
\mathbf{B}_{c 2} & =\left[\begin{array}{l}
1 \\
\mathbf{0}
\end{array}\right], \quad \mathbf{B}_{c 3}=\left[\begin{array}{c}
0 \\
\mathbf{B}_{\Delta}
\end{array}\right], \quad \mathbf{C}_{c}=\left[\begin{array}{ll}
1 & 0
\end{array}\right],
\end{aligned}
$$

where $\mathbf{x}_{c}(t) \in \mathbb{R}^{(m+1) \times 1}, \mathbf{A}_{c} \in \mathbb{R}^{(m+1) \times(m+1)}, \mathbf{B}_{c 1}, \mathbf{B}_{c 2}, \mathbf{B}_{c 3} \in$ $\mathbb{R}^{(m+1) \times 1}$, and $\mathbf{C}_{c} \in \mathbb{R}^{1 \times(m+1)}$.

It is desired that the generator angular speed $\omega_{g}(t)$ accurately tracks the optimal reference trajectory $\omega_{g_{\text {opt }}}(t)$, with tracking error defined by $e_{\omega}(t)=\omega_{g}(t)-\omega_{g_{\text {opt }}}(t)$ absolutely bounded by a small quantity $\varepsilon$; that is, $\sup _{t \geq 0}\left|e_{\omega}(t)\right| \leq \varepsilon$. Then, based on (29), (31), and (35), the following GPI observer-based control is proposed.

Theorem 11. Given Assumptions 9 and 10, the estimation of the perturbation function $\Delta(t)$, denoted as $\widehat{\Delta}(t)$, is given by the following GPI observer:

$$
\begin{aligned}
& \frac{d}{d t} \widehat{\mathbf{x}}_{c}(t)= \mathbf{A}_{c} \widehat{\mathbf{x}}_{c}(t)+\mathbf{B}_{c 1} \tau_{g}(t)+\mathbf{B}_{c 2} \widehat{\varphi}(t) \\
&+\boldsymbol{\sigma}\left(y(t)-\mathbf{C}_{c} \widehat{\mathbf{x}}_{c}(t)\right) \\
& \widehat{\Delta}(t)=\left[\begin{array}{ll}
0 & \mathbf{C}_{\Delta}
\end{array}\right] \widehat{\mathbf{x}}_{c}(t)
\end{aligned}
$$

with,

$$
\begin{aligned}
\widehat{\varphi}(t)= & \frac{B_{d t} \eta_{d t}}{J_{g} N_{g}} \widehat{\omega}_{r}(t)-\frac{B_{g}+\left(B_{d t} \eta_{d t} / N_{g}^{2}\right)}{J_{g}} \widehat{\omega}_{g}(t) \\
& +\frac{K_{d t} \eta_{d t}}{J_{g} N_{g}} \widehat{\theta}_{\Delta}(t),
\end{aligned}
$$

where $\widehat{\mathbf{x}}_{c}(t)=\left[\begin{array}{llllll}\widehat{\omega}_{g}(t) & \widehat{\Delta}(t) & \dot{\widehat{\Delta}}(t) & \cdots & \widehat{\Delta}^{(m-2)}(t) & \widehat{\Delta}^{(m-1)}(t)\end{array}\right]^{\mathbf{T}}$ is the estimated system state vector, $\sigma=$ $\left[\begin{array}{lllll}\sigma_{m+1} & \sigma_{m} & \cdots & \sigma_{2} & \sigma_{1}\end{array}\right]^{T}$ is the observer gains, and $\widehat{\varphi}(t)$ is the estimation of $\varphi(t)$ reconstructed by using the states of the aerodynamic torque observer given in (21) that asymptotically and exponentially reconstructs the perturbation input $\Delta(t)$, forcing the state estimation error $\widetilde{\mathbf{e}}_{\mathbf{x}_{c}}(t)=\mathbf{x}_{c}(t)-\widehat{\mathbf{x}}_{c}(t)$ to converge towards the interior of a disk centered in the origin of the corresponding estimation error phase space, provided the set of coefficients: $\left\{\sigma_{m+1}, \ldots, \sigma_{2}, \sigma_{1}\right\}$, which are chosen in such a way that the polynomial $P_{\Delta}(s)$, in the complex variable $s$, defined by

$$
P_{\Delta}(s)=s^{m+1}+\sigma_{m+1} s^{m}+\cdots+\sigma_{2} s+\sigma_{1}
$$

is a Hurwitz polynomial, with roots located to the left of the imaginary axis of the complex plane.

Proof. By subtracting the proposed GPI observer (37) from the augmented system state equation (35), the following estimation error dynamics is obtained:

$$
\begin{aligned}
\dot{\tilde{\mathbf{e}}}_{\mathbf{x}_{\mathbf{c}}}(t) & =\left(\mathbf{A}_{\mathbf{c}}-\boldsymbol{\sigma} \mathbf{C}_{\mathbf{c}}\right) \widetilde{\mathbf{e}}_{\mathbf{x}_{\mathbf{c}}}(t)+\mathbf{B}_{\mathbf{c} 3} \Delta^{(m)}(t) \\
& =\mathbf{A}_{\mathbf{a c}} \widetilde{\mathbf{e}}_{\mathbf{x}_{\mathbf{c}}}(t)+\mathbf{B}_{\mathbf{c} 3} \Delta^{(m)}(t),
\end{aligned}
$$

where the roots of $\operatorname{det}\left(s \mathbf{I}-\mathbf{A}_{\mathrm{ac}}\right)=s^{m+1}+\sigma_{m+1} s^{m}+\cdots+\sigma_{2} s+$ $\sigma_{1}$ can be placed as desired by selecting the gain vector $\boldsymbol{\sigma}$.

Following the same idea of the proof of Theorem 7, let $\mathbf{Q}_{\mathbf{c}}=\mathbf{I} \in \mathbb{R}^{(m+1) \times(m+1)}$ be a constant, positive definite symmetric matrix; then, a positive definite matrix $\mathbf{P}_{\mathbf{c}} \in$ $\mathbb{R}^{(m+1) \times(m+1)}$ exists such that $\mathbf{A}_{\mathbf{a c}}^{\mathbf{T}} \mathbf{P}_{\mathbf{c}}+\mathbf{P}_{\mathbf{c}} \mathbf{A}_{\mathbf{a c}}=-\mathbf{Q}_{\mathbf{c}}$. Consider the Lyapunov function candidate $V\left(\widetilde{\mathbf{e}}_{\mathbf{x}_{\mathrm{c}}}\right)$ = $(1 / 2) \widetilde{\mathbf{e}}_{\mathbf{x}_{\mathbf{c}}}^{\mathbf{T}}(t) \mathbf{P}_{\mathbf{c}} \widetilde{\mathbf{e}}_{\mathbf{x}_{\mathbf{c}}}(t)$. The time derivative of $V$, that is, $\dot{V}\left(\widetilde{\mathbf{e}}_{\mathbf{x}_{\mathbf{c}}}, t\right)$, is strictly negative outside the disc:

$$
D_{x c}=\left\{\widetilde{\mathbf{e}}_{\mathbf{x}_{\mathbf{c}}}(t) \in \mathbb{R}^{m+1},\left\|\widetilde{\mathbf{e}}_{\mathbf{x}_{\mathbf{c}}}\right\|_{2} \leq 2 K_{\Delta}\left\|\mathbf{P}_{\mathbf{c}}\right\|_{2}\right\} .
$$

Consequently, a uniform ultimate bounded (UUB) result was obtained regarding the estimation error phase variables $\widetilde{\mathbf{e}}_{\mathbf{x}_{\mathrm{c}}}(t)$.

Theorem 12. Assume that there is an accurate estimation of $\varphi(t), \Delta(t), \tau_{a}(t)$, and $\dot{\tau}_{a}(t)$; then, for the simplified system (29), the following control law is proposed:

$$
\tau_{g}(t)=\frac{1}{\kappa}\left[\dot{\widehat{\omega}}_{g_{\mathrm{opt}}}(t)-k_{c}\left(\omega_{g}(t)-\widehat{\omega}_{g_{\mathrm{opt}}}(t)\right)-\widehat{\varphi}(t)-\widehat{\Delta}(t)\right]
$$


with

$$
\dot{\widehat{\omega}}_{g_{\mathrm{opt}}}(t)=\frac{N_{g} \lambda_{\mathrm{opt}}}{2 R} \sqrt{\frac{2 \lambda_{\mathrm{opt}}}{\rho \pi R^{3} C_{p_{\mathrm{opt}}}}} \frac{1}{\sqrt{\widehat{\tau}_{a}(t)}} \dot{\hat{\tau}}_{a}(t),
$$

where $\hat{\tau}_{a}(t)$ and $\dot{\hat{\tau}}_{a}(t)$ are provided by the aerodynamic torque observer given in (21), $\widehat{\varphi}(t)$ is reconstructed by the estimated states of the aerodynamic torque observer given in (21), and $\widehat{\Delta}(t)$ is provided by the GPI disturbance observer given in (37) and (38).

Such law asymptotically and exponentially forces the closed loop system tracking error $e_{\omega}(t)$ to converge towards the interior of a disk of radius as small as desired centered in zero, provided that the coefficient is $k_{c}>0$.

Proof. By replacing (43) in (29), the following dynamics is obtained:

$$
\begin{gathered}
\dot{\omega}_{g}(t)-\dot{\hat{\omega}}_{g_{\mathrm{opt}}}(t)+k_{c}\left(\omega_{g}(t)-\widehat{\omega}_{g_{\mathrm{opt}}}(t)\right) \\
=-\widehat{\varphi}(t)-\widehat{\Delta}(t)+\Delta(t)+\varphi(t) .
\end{gathered}
$$

Then, by defining some estimation errors: $\widetilde{e}_{\omega_{\text {opt }}}=\omega_{g_{\text {opt }}}(t)-$ $\widehat{\omega}_{g_{\text {opt }}}(t), \widetilde{e}_{\Delta}(t)=\Delta(t)-\widehat{\Delta}(t)$, and $\tilde{e}_{\varphi}(t)=\varphi(t)-\widehat{\varphi}(t)$ and by replacing them in (45), the following control system tracking error dynamics is obtained:

$$
\begin{gathered}
\left(\dot{\omega}_{g}(t)-\dot{\omega}_{g_{\mathrm{opt}}}(t)\right)+k_{c}\left(\omega_{g}(t)-\omega_{g_{\mathrm{opt}}}(t)\right) \\
=-\dot{\tilde{e}}_{\omega_{\mathrm{opt}}}(t)-k_{c} \widetilde{e}_{\omega_{\mathrm{opt}}}(t)+\widetilde{e}_{\Delta}(t)+\widetilde{e}_{\varphi}(t), \\
\dot{e}_{\omega}(t)+k_{c} e_{\omega}(t)=-\dot{\tilde{e}}_{\omega_{\mathrm{opt}}}(t)-k_{c} \widetilde{e}_{\omega_{\mathrm{opt}}}(t)+\widetilde{e}_{\Delta}(t)+\widetilde{e}_{\varphi}(t) .
\end{gathered}
$$

Therefore, as long as $k_{c}>0$ and the estimation errors $\widetilde{e}_{\omega_{\text {opt }}}(t)$, $\tilde{e}_{\Delta}(t)$, and $\tilde{e}_{\varphi}(t)$ are ultimately bounded by the GPI observers (21) and (37), the tracking error dynamics $e_{\omega}(t)$ will remain stable and bounded since the right side of (46) is also bounded.

Since the relative order of the WECS is one and system order is three, zero dynamics come into play and in consequence are analyzed. Considering the third-order system dynamics defined in (7), the zero dynamics is given by $\omega_{r}(t)$ and $\theta_{\Delta}(t)$ with $\omega_{g}(t)$ set to zero:

$$
\left[\begin{array}{c}
\dot{\omega}_{r}(t) \\
\dot{\theta}_{\Delta}(t)
\end{array}\right]=\underbrace{\left[\begin{array}{cc}
-\frac{B_{d t}+B_{r}}{J_{r}} & -\frac{K_{d t}}{J_{r}} \\
1 & 0
\end{array}\right]}_{A_{\text {zero }}}\left[\begin{array}{c}
\omega_{r}(t) \\
\theta_{\Delta}(t)
\end{array}\right]+\left[\begin{array}{c}
\frac{1}{J_{r}} \\
0
\end{array}\right] \tau_{a}(t) .
$$

Then, the internal dynamics is now given by the eigenvalues of $A_{\text {zero }}$ (48). In this work, the eigenvalues are stable since the parameters of the WECS $B_{d t}, B_{r}, K_{d t}$, and $J_{r}$ are positive:

$$
\left|s I-A_{\text {zero }}\right|=\left|\begin{array}{cc}
s+\frac{B_{d t}+B_{r}}{J_{r}} & \frac{K_{d t}}{J_{r}} \\
-1 & s
\end{array}\right|=s^{2}+\frac{B_{d t}+B_{r}}{J_{r}} s+\frac{K_{d t}}{J_{r}} .
$$

Some troubles may arise in the control system when the internal dynamics is poorly damped; however, in the studied case, such internal dynamics does not lead to any problem. In cases where the internal dynamics became problematic, some methods are used [31].

Remark 13. Note that for the GPI observer-based control strategy defined in (43) and (37), the energy capture maximization depends on the accurate reconstruction of the optimal reference trajectory $\omega_{g_{\mathrm{opt}}}(t)$.

\section{Benchmark Model}

The simulations are carried out using a benchmark model for wind turbine control implemented in MATLAB/Simulink. This benchmark model was published by Odgaard et al. in [25] and can be used to evaluate both fault tolerant and classic control schemes in any region of operation of a wind turbine. The test bench model is based on a realistic nonlinear generic three-bladed horizontal-axis variable-speed wind turbine, containing sensors, actuators, system faults, tower shadow and wind shear effects, full converter coupling, and rated power at $4.8 \mathrm{MW}$.

For wind speeds between 0 and $12.5 \mathrm{~m} / \mathrm{s}$, the turbine is controlled to operate in region 2 . The wind profile used has an average hub-height wind speed of $8.68 \mathrm{~m} / \mathrm{s}$ and a turbulence intensity of $12 \%$. The test bench defines a standard torque control strategy for the operation in region 2 with the following control law: $\tau_{g, \text { ref }}(t)=k_{\text {opt }} \omega_{g}^{2}(t)$ with $k_{\text {opt }}=1.2171, C_{p_{\text {opt }}}=$ 0.4554 , and $\lambda_{\text {opt }}=8.0$. The converter model has the following constraints: $\max$ torque gradient $=1.25 \times 10^{4} \mathrm{~N} \cdot \mathrm{m} / \mathrm{s}$, $\min$ torque gradient $=-1.25 \times 10^{4} \mathrm{~N} \cdot \mathrm{m} / \mathrm{s}, \max$ torque $=3.6 \times$ $10^{4} \mathrm{~N} \cdot \mathrm{m}$, and $\min$ torque $=0 \mathrm{~N} \cdot \mathrm{m}$.

\section{Results and Discussion}

The proposed aerodynamic torque GPI observer (21) and the GPI observer-based control (37)-(43) were implemented and tested on the nonlinear wind turbine benchmark model described previously. Figure 2 shows the system scheme of the proposed control strategy used for simulations. The parameters of the GPI observers and the control strategy were selected as follows:

GPI observer for aerodynamic torque estimation: $m=3, \sigma_{1}=1000$, and $\sigma_{2}=1028.22, \sigma_{3}=1028.62$, $\sigma_{4}=0.39, \sigma_{5}=234.81, \sigma_{6}=28.46$.

GPI observer-based control: $p=3, k_{c}=1, \alpha_{1}=$ $39.38, \alpha_{2}=61.69, \alpha_{3}=35.30$, and $\alpha_{4}=8.66$.

5.1. Aerodynamic Torque Estimation. The estimate of the aerodynamic torque obtained by the GPI observer in (21) is shown in Figure 3(a). The aerodynamic torque estimation error is shown in Figure 3(c). The estimation of the generator speed optimal reference trajectory $\widehat{\omega}_{g_{\text {opt }}}(t)$ is shown in Figure $3(\mathrm{~b})$, and its estimation error $\widetilde{e}_{\omega_{\mathrm{opt}}}=\omega_{g_{\mathrm{opt}}}(t)-\widehat{\omega}_{g_{\mathrm{opt}}}(t)$ can be detailed in Figure 3(d). Note that this estimation error 


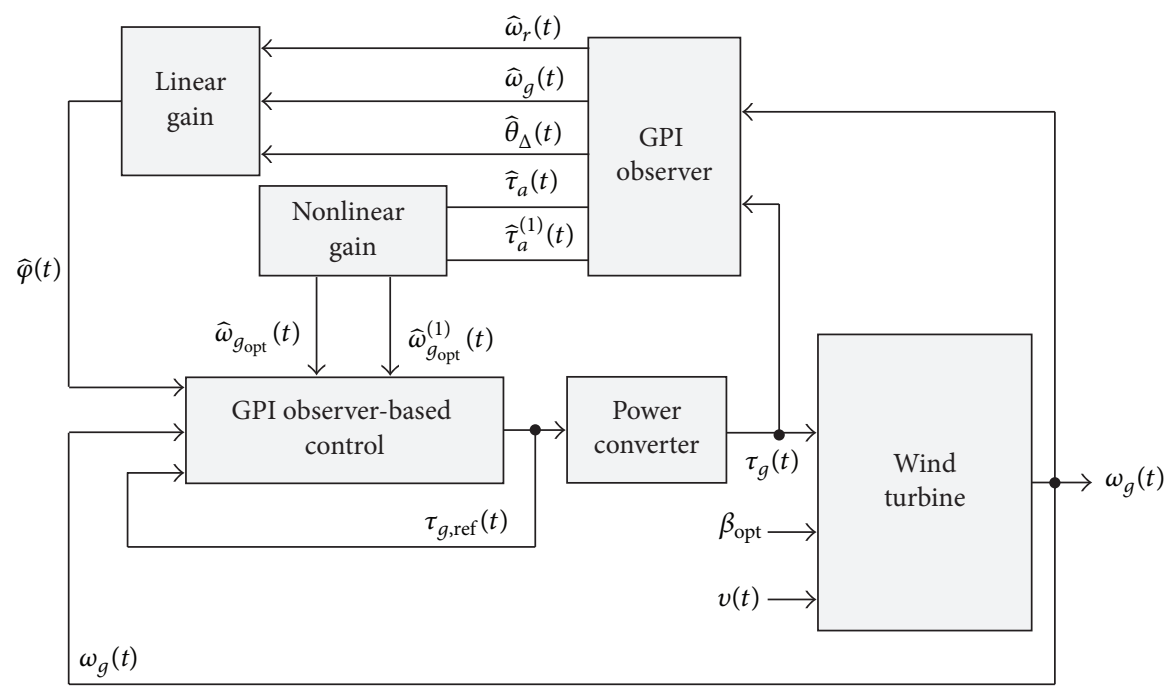

Figure 2: Closed loop system schema of the proposed control strategy.

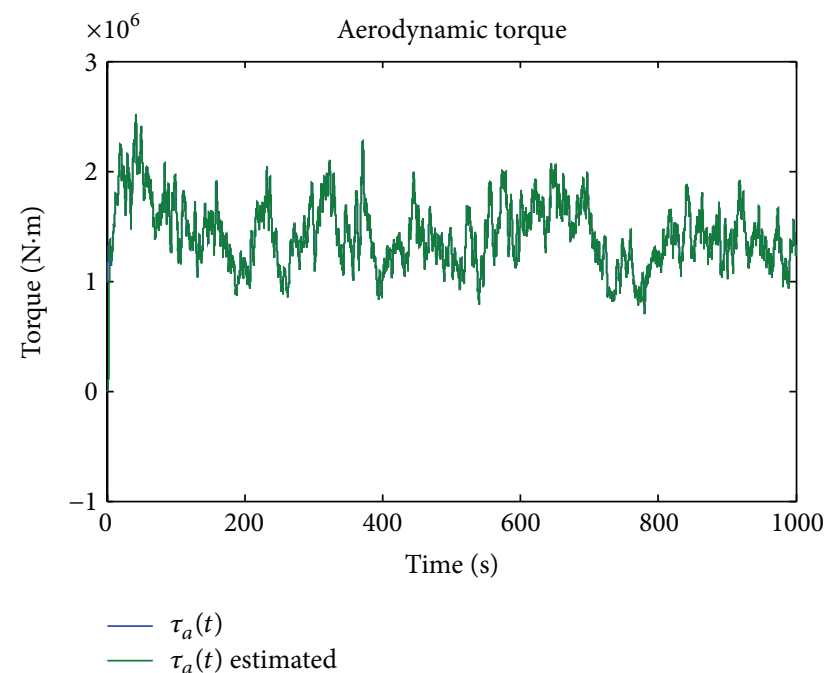

(a)

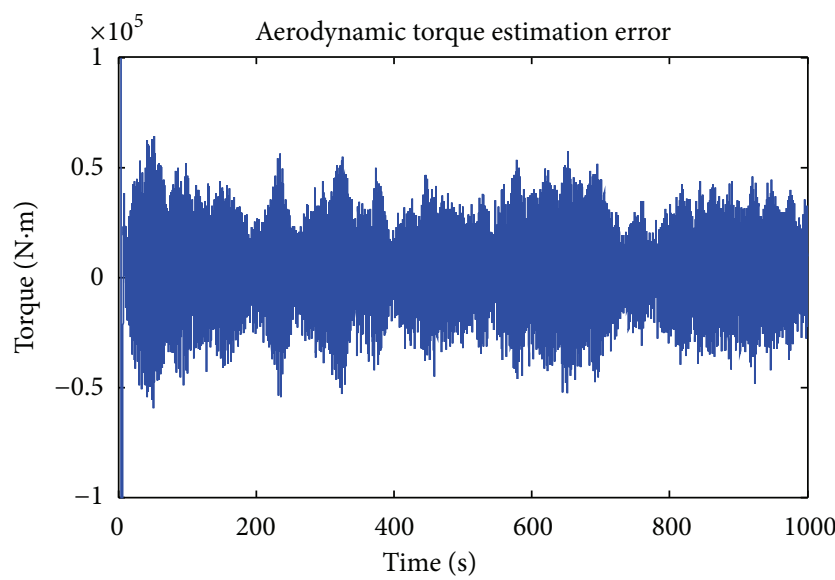

(c)

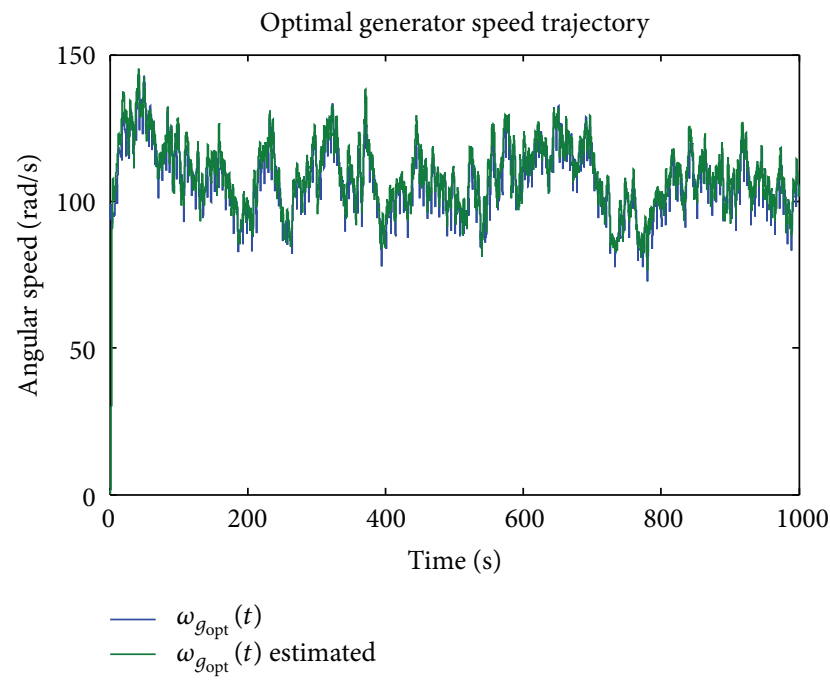

(b)

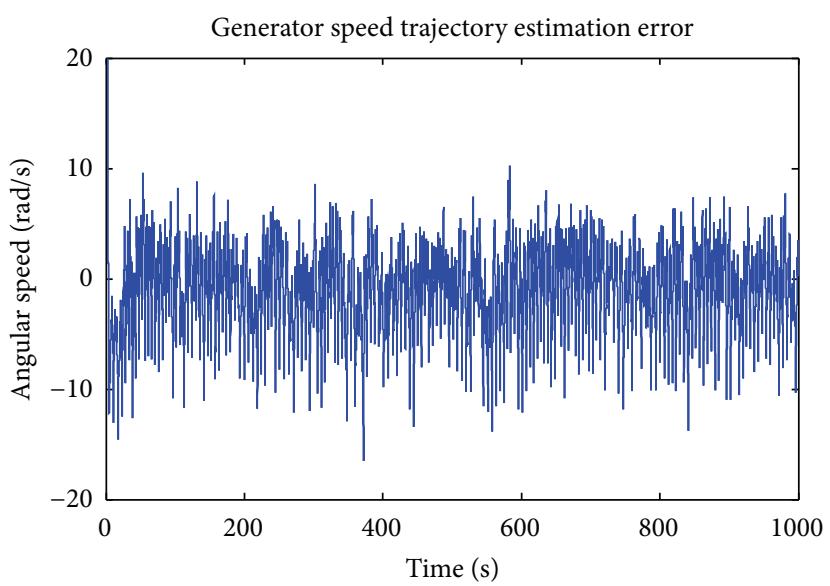

(d)

Figure 3: Aerodynamic torque estimation results: (a) aerodynamic torque, (b) generator speed optimal trajectory, (c) aerodynamic torque estimation error, and (d) generator speed trajectory estimation error. 


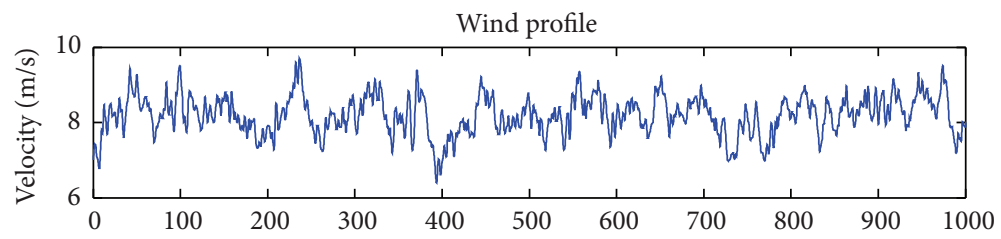

(a)

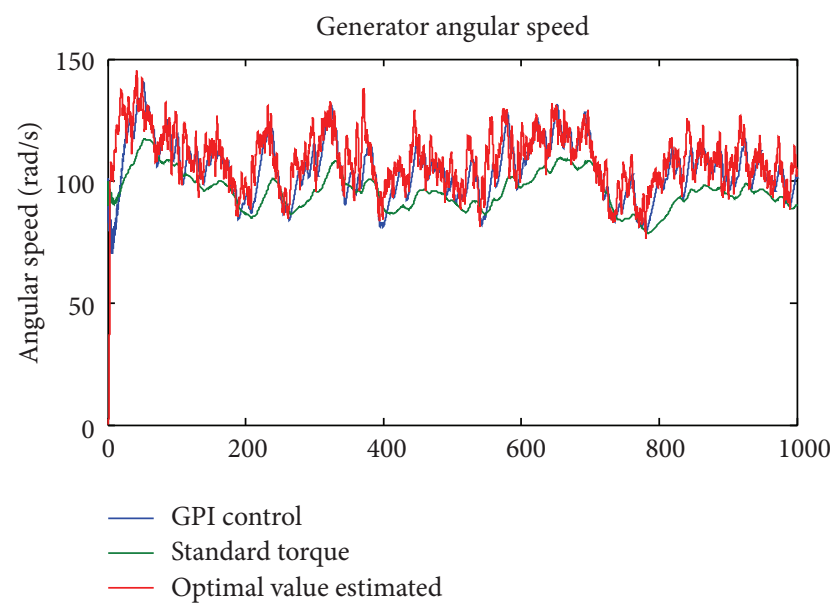

(b)

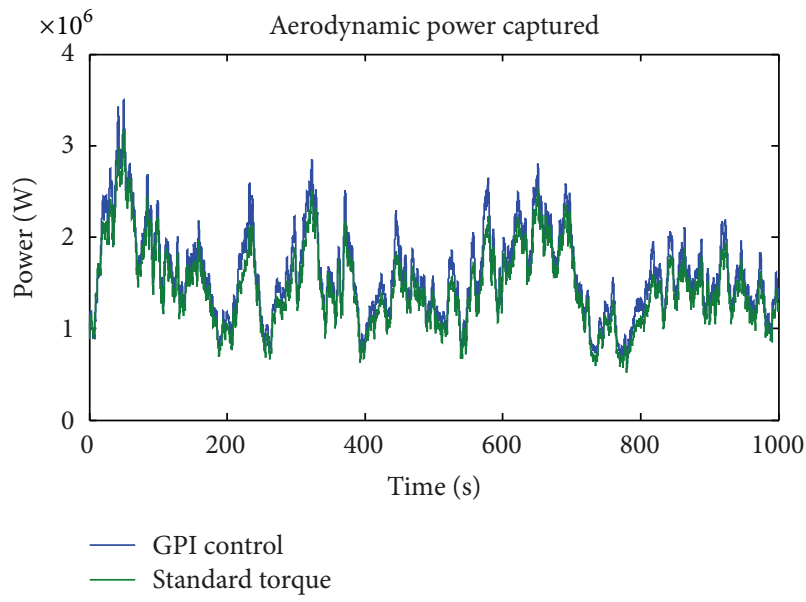

(d)

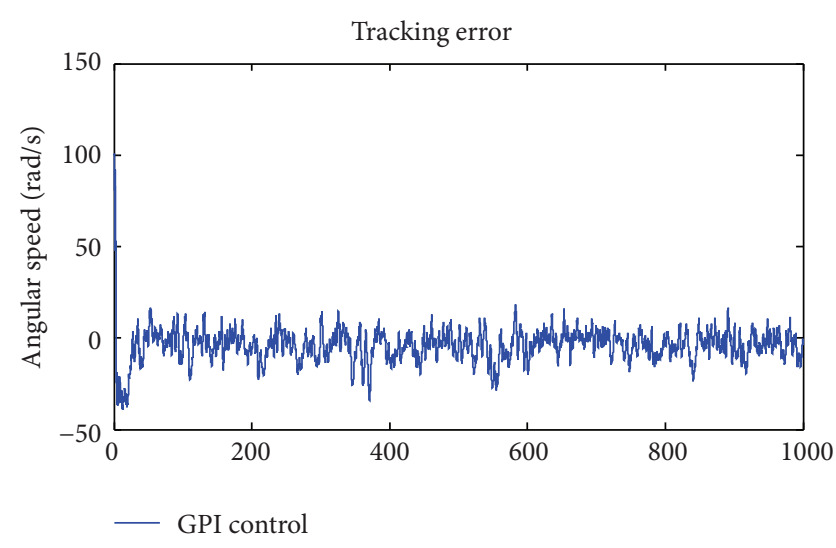

(c)

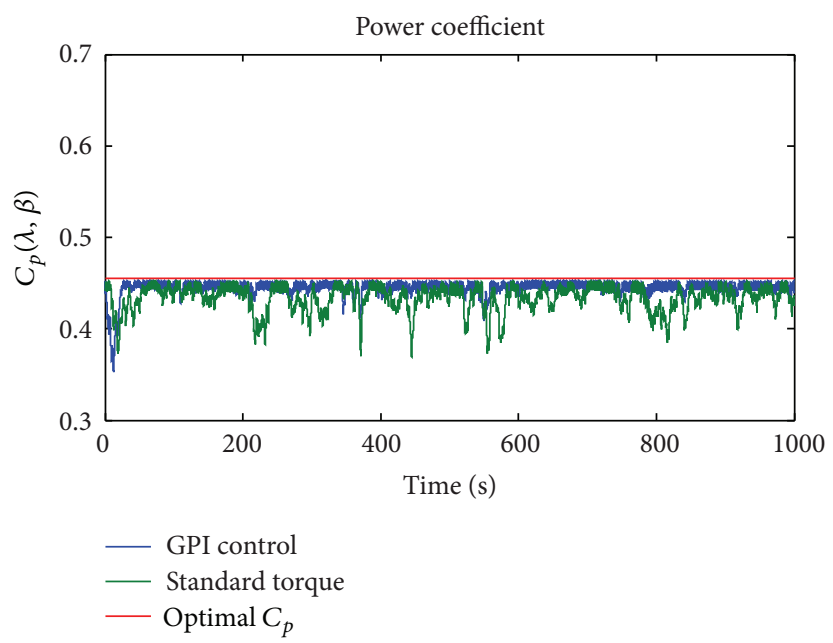

(e)

FiguRE 4: Simulation results of the proposed GPI observer-based control: (a) wind profile, (b) generator angular speed, (c) tracking error, (d) aerodynamic power captured, and (e) power coefficient.

has a small offset of $-1.0811 \mathrm{rad} / \mathrm{s}$, which in turn will cause a slight offset in the aerodynamic power coefficient control.

5.2. Energy Capture Maximization. The simulation results of the proposed control strategy are shown in Figure 4. Under nominal conditions, the control system tracks the optimal reference trajectory to force the WECS power coefficient $C_{p}$ close to its optimal value (see Figures 4(b) and $4(\mathrm{e})$ ). Despite the external disturbances and nonlinearities of the benchmark model, the tracking error of the control system is near to zero as shown in Figure 4(c). In order to achieve a compromise between energy capture and dynamic loads on the low speed shaft, a medium performance on the control gain $k_{c}$ was selected. Most of the fast fluctuations of the aerodynamic torque transferred to the generator speed optimal trajectory are not tracked (see Figure 4(b)).

Figures 4(d) and 4(e) detail the aerodynamic power captured by the GPI observer-based control and the evolution in time of the WECS power coefficient, respectively. It is observed that the captured aerodynamic power with the proposed control is greater than the power captured by the standard torque control defined in the benchmark. In addition, it is noted that the proposed control strategy forces the power coefficient to stay close to its optimal value $C_{p_{\mathrm{opt}}}=$ 0.4554 , which allows better power capture. 


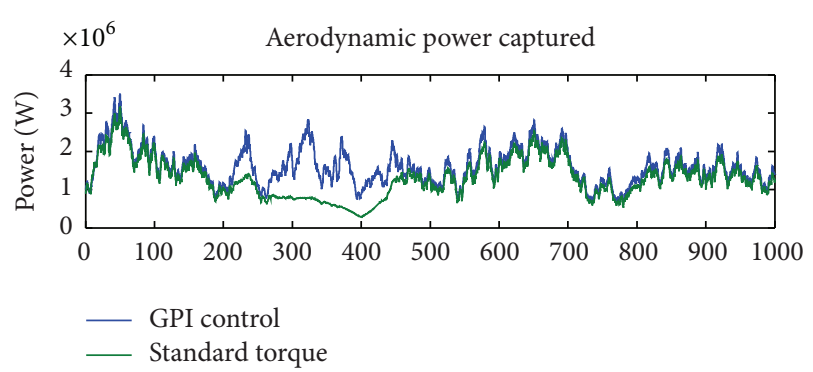

(a)

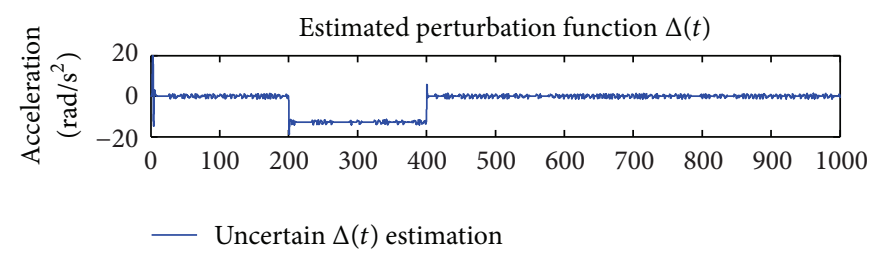

(c)

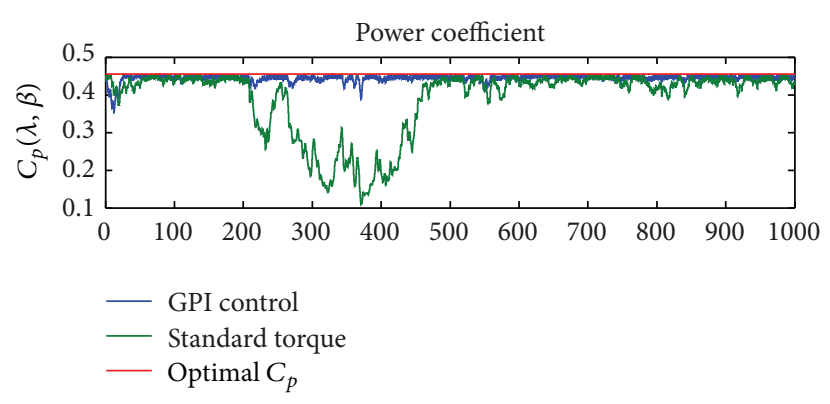

(b)

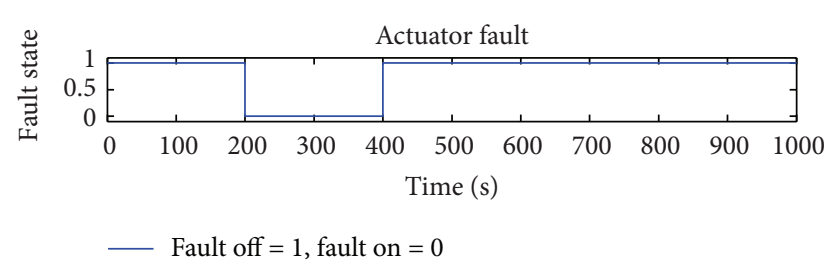

(d)

FIGURE 5: Simulation results on power converter fault.

The performance of each control system is compared using an aerodynamic efficiency index $\eta_{\text {aero }}$ [24]. It is defined as follows:

$$
\eta_{\text {aero }}(\%)=\frac{\int_{t_{i}}^{t_{f}} P_{a}(t) d t}{\int_{t_{i}}^{t_{f}} P_{a_{\mathrm{opt}}}(t) d t}, \quad P_{a_{\mathrm{opt}}}(t)=\frac{1}{2} \rho \pi R^{2} C_{p_{\mathrm{opt}}} v(t)^{3} .
$$

The evaluation of the criteria defined in (49) stated that the aerodynamic efficiency obtained by the proposed control technique is $98.8 \%$, while the efficiency of the classical controller (benchmark) is $95.6 \%$.

5.3. Active Disturbance Rejection Evaluation. The benchmark model [25] contains faults which require the control system to be reconfigured to continue power generation, as well as very severe faults which require a safe and fast shutdown of the wind turbine. In this work, in order to evaluate the active disturbance rejection capability of the proposed GPI observerbased control strategy, a typical malfunction in the internal power converter control loops is used. As a consequence, this nonsevere fault must be accommodated in some way, and the wind turbine must continue its operation.

The fault considered is an offset, denoted as $\Delta \tau_{g}$, on the generator torque, which can be caused by an error in the initialization of the converter controller [32]. The converter offset is configured to $\Delta \tau_{g}=5000 \mathrm{~N} \cdot \mathrm{m}$.

Figure 5 shows the closed loop performance of both the standard torque control and the proposed GPI observerbased control approach under the actuator fault. The fault occurs from $200 \mathrm{~s}$ to $400 \mathrm{~s}$ as seen in Figure 5(d). As the fault is active, the GPI observer (37), via the observer state $\widehat{\Delta}(t)$, estimates the perturbation function on line (see Figure 5(c)) and actively rejects the internal perturbation applying the GPI observer-based control law (43).
It is observed in Figure 5(a) that the perturbation is rejected by the proposed control and any captured aerodynamic power lost is appreciated. Figure 5(b) shows that the power coefficient of the WECS is still close to its optimal value. On the other hand, the standard torque control of the benchmark cannot handle the actuator fault, and much of the aerodynamic power is lost. The evaluation of the criteria defined in (49) stated that the aerodynamic efficiency obtained by the proposed control is $98.65 \%$, while the efficiency of the classical controller (benchmark) is $85.88 \%$.

\section{Conclusions}

In this paper, a linear active disturbance rejection control strategy based on two GPI observers for maximum wind energy capture of variable-speed wind turbines operating at partial load has been proposed. In order to create the generator speed optimal trajectory towards an optimum point at which the WECS power coefficient is maximum, an ADR philosophy-based GPI observer was developed to estimate the aerodynamic torque and its first derivative. Then, an ADR philosophy-based GPI observer-based controller was designed, and it was able to absolutely and arbitrarily bound the generator speed tracking error.

The proposed design strategy solved the control problem based on linear active estimation of possible nonlinearities and perturbations of the WECS, and these accurate estimations were used by a simplified linear control law, in which the captured wind energy was maximized.

It was shown through simulation tests on a fully nonlinear benchmark model that the proposed dual GPI observer control strategy maximized the captured wind energy even when an actuator fault was applied. This is a demonstration of some robustness added by the GPI observer-based control. 
Since performance of wind turbines is significantly affected by the used control strategy, considering new alternative linear control strategies that can improve the performance of the WECS is a motivation to use, adapt, and evaluate linear GPI observer-based controllers to operate wind turbines at partial load.

It is worth noting that the proposed control strategy is related to exact feedback linearization, but there are some important differences between both strategies which give advantages to GPI observer-based control, such as the following: (a) GPI observer-based control does not require system state measurements, (b) any mismatch between the system model and the real system is lumped together in a perturbation function that is estimated and rejected on line, (c) GPI observers are capable of estimating a certain number of perturbation function derivatives (useful to determine $\dot{\omega}_{g_{\text {opt }}}$ ), and (d) ADR philosophy plays a very important role in GPI observer-based control since the internal model of the perturbation functions is taken into account in the design process.

\section{References}

[1] I. Munteanu, A. I. Bratcu, N.-A. Cutululis, and E. Ceanga, Optimal Control of Wind Energy Systems, Springer, London, UK, 2008.

[2] F. D. Bianchi, R. S. Sánchez-Peña, and M. Guadayol, "Gain scheduled control based on high fidelity local wind turbine models," Renewable Energy, vol. 37, no. 1, pp. 233-240, 2012.

[3] G. A. Parker and C. D. Johnson, "Improved speed regulation and mitigation of drive-train torsion fatigue in flexible wind turbines, using disturbance utilization control: part one," in Proceedings of the 41st Southeastern Symposium on System Theory (SSST '09), pp. 171-176, March 2009.

[4] L. Y. Pao and K. E. Johnson, "Control of wind turbines: approaches, challenges, and recent developments," IEEE Control Systems Magazine, vol. 31, no. 2, pp. 44-62, 2011.

[5] E. Iyasere, M. Salah, D. Dawson, J. Wagner, and E. Tatlicioglu, "Optimum seeking-based non-linear controller to maximise energy capture in a variable speed wind turbine," IET Control Theory \& Applications, vol. 6, no. 4, pp. 526-532, 2012.

[6] K. Pierce, "Control Method for Improved Energy Capture below Rated Power," National Renewable Energy Laboratory NREL/CP-500-26322, 1999.

[7] K. A. Stol, "Disturbance tracking control and blade load mitigation for variable-speed wind turbines," Journal of Solar Energy Engineering, Transactions of the ASME, vol. 125, no. 4, pp. 396-401, 2003.

[8] W. E. Leithead and B. Connor, "Control of variable speed wind turbines: design task," International Journal of Control, vol. 73, no. 13, pp. 1189-1212, 2000.

[9] H. Vihriälä, R. Perälä, P. Mäkilä, and L. Söderlund, "A gearless wind power drive: part 2: performance of control system," in Proceedings of the European Wind Energy Conference, , pp. 10901093, 2001.

[10] C. Evangelista, P. Puleston, F. Valenciaga, and A. Dávila, "Variable gains super-twisting control for wind energy conversion optimization," in Proceedings of the 11th International Workshop on Variable Structure Systems (VSS '10), pp. 50-55, June 2010.

[11] B. Beltran, M. E. H. Benbouzid, and T. Ahmed-Ali, "Secondorder sliding mode control of a doubly fed induction generator driven wind turbine," IEEE Transactions on Energy Conversion, vol. 27, pp. 261-269, 2012.

[12] C. Evangelista, P. Puleston, F. Valenciaga, and L. M. Fridman, "Lyapunov-designed super-twisting sliding mode control for wind energy conversion optimization," IEEE Transactions on Industrial Electronics, vol. 60, pp. 538-545, 2013.

[13] M. Fliess, R. Marquez, E. Delaleau, and H. Sira-Ramírez, "Correcteurs proportionnels-intégraux généralisés," ESAIM. Control, Optimisation and Calculus of Variations, vol. 7, pp. 23-41, 2002.

[14] A. Luviano-Juárez, J. Cortés-Romero, and H. Sira-Ramírez, "Synchronization of chaotic oscillators by means of generalized proportional integral observers," International Journal of Bifurcation and Chaos in Applied Sciences and Engineering, vol. 20, no. 5, pp. 1509-1517, 2010.

[15] H. Sira-Ramírez and V. F. Batlle, "Robust $\Sigma-\Delta$ modulationbased sliding mode observers for linear systems subject to time polynomial inputs," International Journal of Systems Science, vol. 42, no. 4, pp. 621-631, 2011.

[16] M. Fliess, R. Marquez, and E. Delaleau, "State feedbacks without asymptotic observers and generalized PID regulators," in Nonlinear Control in the Year 2000, A. Isidori, F. LamnabhiLagarrigue, and W. Respondek, Eds., vol. 258, pp. 367-384, Springer, Berlin, Germany, 2001.

[17] H. Sira-Ramírez, F. González-Montañez, J. Cortés-Romero, and A. Luviano-Juarez, "A disturbance rejection approach for the induction motor through observer based Generalized PI control," in Proceedings of the American Control Conference, Fairmont Queen Elizabeth, Montréal, Canada, 2012.

[18] H. Sira-Ramírez, A. Luviano-Juárez, and J. Cortés-Romero, "Flatness-based linear output feedback control for disturbance rejection and tracking tasks on a Chua's circuit," International Journal of Control, vol. 85, no. 5, pp. 594-602, 2012.

[19] H. Sira-Ramírez, A. Luviano-Juárez, and J. Cortés-Romero, "Robust input-output sliding mode control of the buck converter," Control Engineering Practice, vol. 21, pp. 671-678, 2013.

[20] C. D. Johnson, "Accommodation of external disturbances in linear regulator and servomechanism problems," IEEE Transactions on Automatic Control, vol. 16, no. 6, pp. 635-644, 1971.

[21] J. Han, "From PID to active disturbance rejection control," IEEE Transactions on Industrial Electronics, vol. 56, no. 3, pp. 900906, 2009.

[22] G. Tian and Z. Gao, "From poncelet's invariance principle to active disturbance rejection," in Proceedings of the American Control Conference (ACC '09), pp. 2451-2457, June 2009.

[23] Z. Gao, Y. Huang, and J. Han, "An alternative paradigm for control system design," in Proceedings of the 40th IEEE Conference on Decision and Control (CDC '01), pp. 4578-4585, December 2001.

[24] B. Boukhezzar and H. Siguerdidjane, "Nonlinear control of a variable-speed wind turbine using a two-mass model," IEEE Transactions on Energy Conversion, vol. 26, no. 1, pp. 149-162, 2011.

[25] P. F. Odgaard, J. Stoustrup, and M. Kinnaert, "Fault tolerant control of wind turbines-a benchmark model," in Proceedings of the 7th IFAC Symposium on Fault Detection, Supervision and Safety of Technical Processes, pp. 155-160, Barcelona, Spain, July 2009.

[26] G. A. Parker and C. D. Johnson, "Improved speed regulation and mitigation of drive-train torsion fatigue in flexible wind turbines, using disturbance utilization control: part two," in 
Proceedings of the 41st Southeastern Symposium on System Theory (SSST '09), pp. 177-183, March 2009.

[27] L. B. Freidovich and H. K. Khalil, "Performance recovery of feedback-linearization-based designs," IEEE Transactions on Automatic Control, vol. 53, no. 10, pp. 2324-2334, 2008.

[28] S. Zhao and Z. Gao, "An active disturbance rejection based approach to vibration suppression in two-inertia systems," in Proceedings of the American Control Conference (ACC '10), pp. 1520-1525, July 2010.

[29] S. Zhao, Q. Zheng, and Z. Gao, "On model-free accommodation of actuator nonlinearities," in Proceedings of the 10th World Congress on Intelligent Control and Automation, Beijing, China, 2012.

[30] Q. Zheng, Z. Chen, and Z. Gao, "A practical approach to disturbance decoupling control," Control Engineering Practice, vol. 17, no. 9, pp. 1016-1025, 2009.

[31] C. Sloth, T. Esbensen, and J. Stoustrup, "Robust and faulttolerant linear parameter-varying control of wind turbines," Mechatronics, vol. 21, no. 4, pp. 645-659, 2011.

[32] P. F. Odgaard and K. E. Johnson, "Wind turbine fault detection and fault tolerant control-a second challenge," in Proceedings of the 8th IFAC Symposium on Fault Detection, Supervision and Safety of Technical Processes, 2012. 


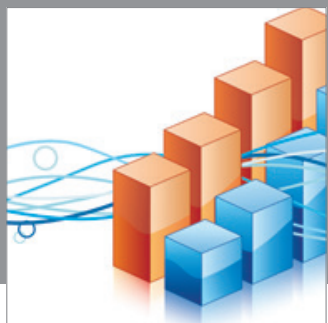

Advances in

Operations Research

mansans

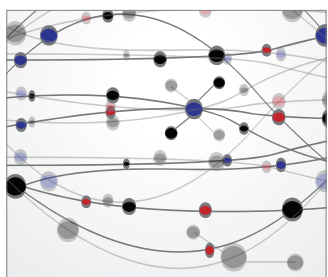

The Scientific World Journal
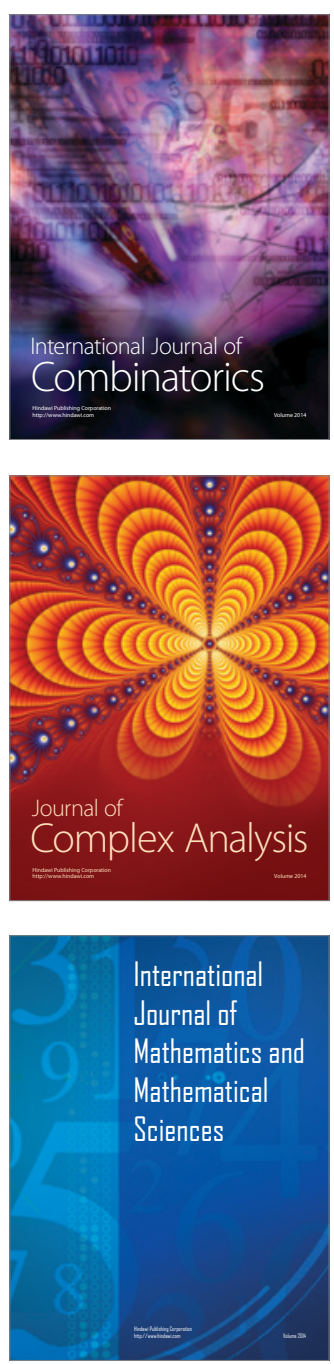
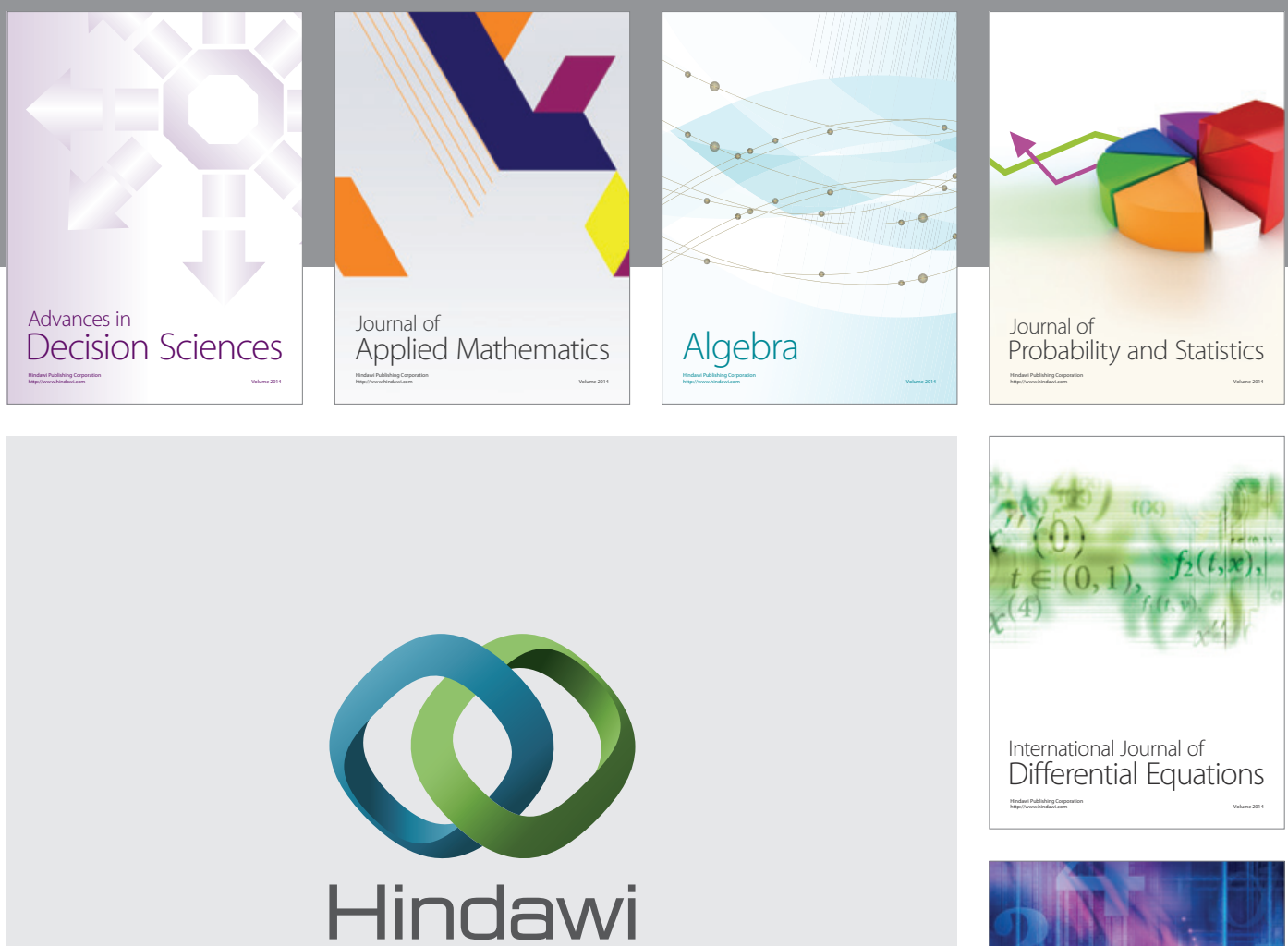

Submit your manuscripts at http://www.hindawi.com
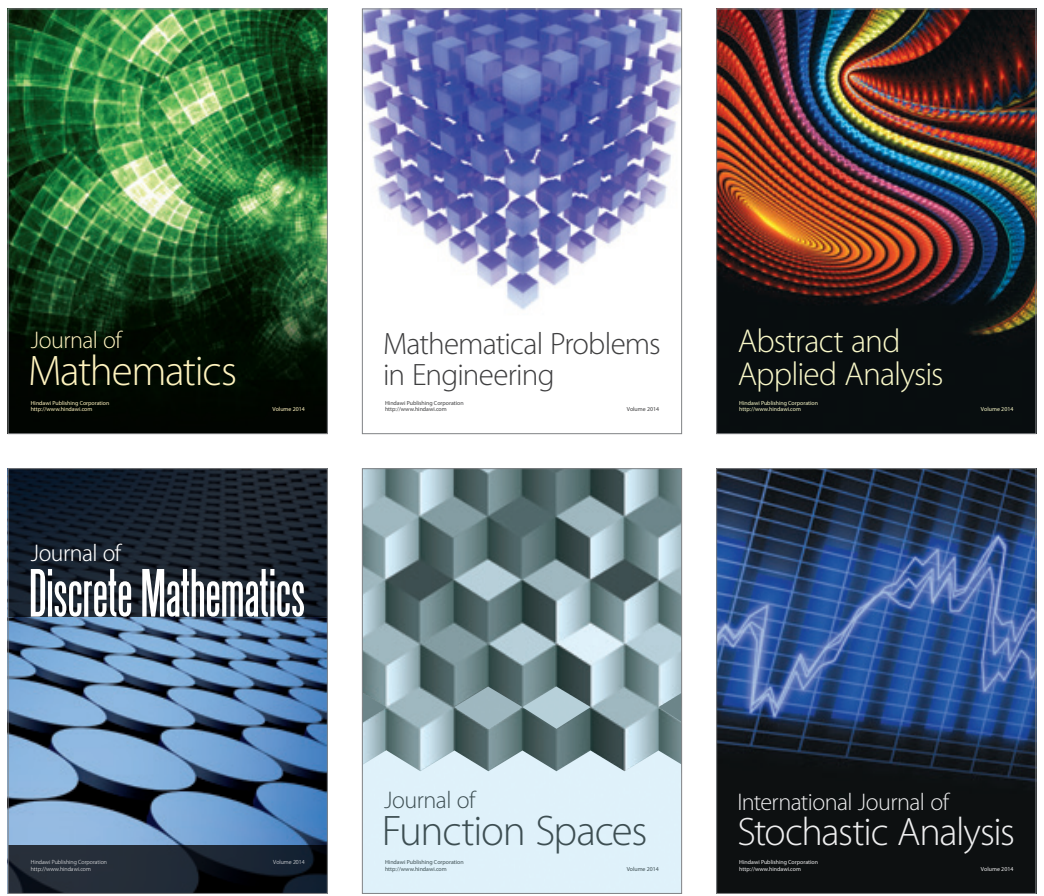

Journal of

Function Spaces

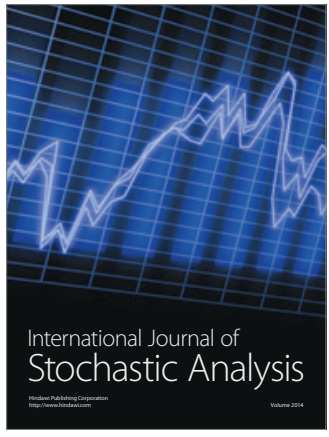

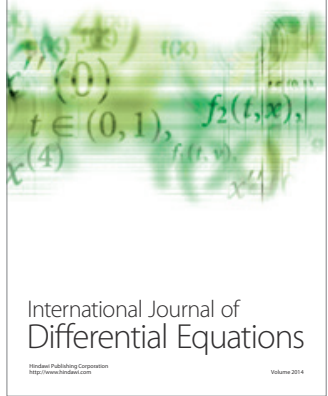
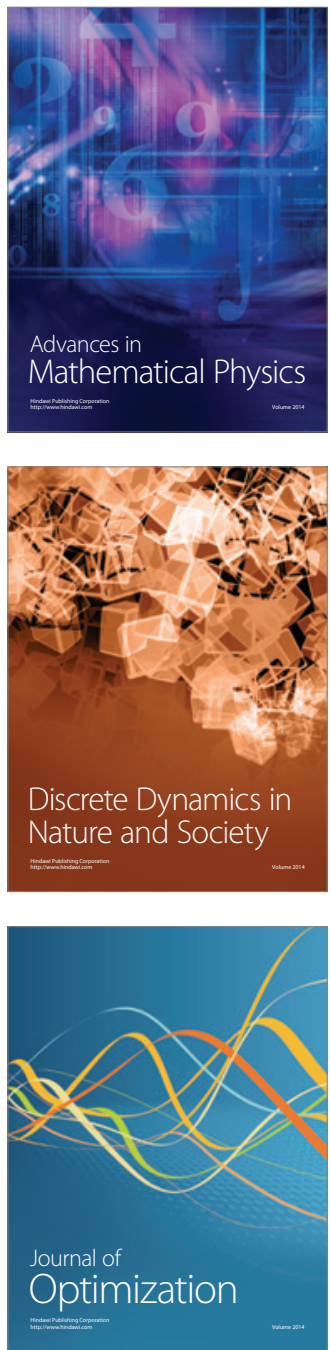